\title{
Inductive Learning of Simple Diagnostic Scores
}

\author{
Martin Atzmueller, Joachim Baumeister, and Frank Puppe \\ University of Würzburg, 97074 Würzburg, Germany \\ Department of Computer Science \\ Phone: +49 931 888-6746, Fax: +49 931 888-6732 \\ email: \{atzmueller, baumeister, puppe\}@informatik.uni-wuerzburg.de
}

\begin{abstract}
Knowledge acquisition and maintenance in medical domains with a large application domain ontology is a difficult task. To reduce knowledge elicitation costs, semi-automatic learning methods can be used to support the expert. We propose diagnostic scores as a promising approach and present a method for inductive learning of diagnostic scores. It can be be refined incrementally by applying different types of background knowledge. We give an evaluation of the presented approach with a real-world case base.
\end{abstract}

\section{Introduction}

Constructing and in particular maintaining a knowledge base in medical domains is a difficult task. If the degree of connectivity between findings and diagnoses is potentially high, then managing the sheer number of relations is a major problem. Pure automatic learning method are usually not good enough to reach a quality comparable to manually built knowledge bases. However, they can be used to support the expert. In such semi-automatic scenarios, understandability and interpretability of the learned models is of prime importance. Ideally, the learning method constructs knowledge in the same representation the human expert favors.

A rather wide spread formalism for medical decision making are diagnostic scores, e.g. [12]. For inferring a concept, a limited number of features is used in a regular and simple to interpret manner, which can be applied even without a computer. In its simplest form, each feature - if observed in a case - individually contributes one point to an account (score), and if the score exceeds a threshold, the concept is established. Variations concern using several categories instead of one point, acquiring both negative and positive contributions, and utilizing several thresholds to express different degrees, e.g. to differentiate between "possible" and "probable" for inferring a concept.

In comparison to general rules, scores usually have no logical combinations in the precondition. Compared to Bayesian nets, scores have much simpler relations than the probability tables. Similar to both, they can be arranged hierarchically, i.e. a concept inferred with a score can be used to infer another concept. Of course, scores can be refined into both formalisms, but are quite expressable in itself. We therefore propose diagnostic scores as a promising approach to support knowledge engineering. Thus starting with automatically learned knowledge, the expert is able to use the learned knowledge as a starting point in constructing a knowledge base, such that the knowledge can be refined, tuned and extended as needed. One measure for the quality of a score 
is the accuracy of inferring the respective concept. A second important criterion is the complexity of the score, e.g. depending on the number of features used for the score. We present an inductive method for learning diagnostic scores paying special attention to compromise between both criteria. The method can be refined incrementally using different types of background knowledge, which improve the learned scores.

Our implementation and evaluation is based on the knowledge-based documentation and consultation system for sonography SONOCONSULT (an advanced and isolated part of HepatoConsult [3]) based on the diagnostic shell kit D3 [4]. This system is in routine use in the DRK-hospital in Berlin/Köpenick. The cases are detailed descriptions of findings of the examination(s), together with the inferred diagnoses (concepts). Both observations and diagnoses may be ordered hierarchically according to a specialization hierarchy from general to more detailed elements. This setting yields a high quality of the case base with detailed and usually correct case descriptions.

The rest of the paper is organized as follows: In Section 2 we introduce the basic notions, diagnostic scores and their basic properties. In Section 3 we first give essential basic definitions for the general learning task. Then we describe the method of learning diagnostic scores, and discuss which additional knowledge can be applied and its effect. An evaluation with a real-world case base is given in Section 4 . We will conclude the paper in Section 5 with a discussion of the presented work, and we show promising directions for future work.

\section{Basic Definition and Notions}

In the following we define necessary notions that are considered for the learning task. The basic definitions for this task are the following:

We define $\Omega_{Q}$ to be the universe of of all questions available in the problem domain. In context of machine learning methods, questions are commonly called attributes. The type of a question $q \in \Omega_{Q}$ depends on the value range $\operatorname{dom}(q)$. The value range can consist of numerical or symbolic values. A question $q \in \Omega_{Q}$ assigned to a value $v \in$ $\operatorname{dom}(q)$ is called a finding and we call $\Omega_{\mathcal{F}}$ the set of all possible findings in the given problem domain. A finding $f \in \Omega_{\mathcal{F}}$ is denoted by $q: v$ for $q \in \Omega_{Q}$ and $v \in \operatorname{dom}(q)$.

Let $d$ be a diagnosis representing a possible solution and let $\Omega_{\mathcal{D}}$ be the universe of all possible diagnoses for a given problem domain. A diagnosis $d \in \Omega_{\mathcal{D}}$ is assigned to a symbolic state $\operatorname{dom}(d)=\{$ excluded, unclear, probable $\}$ with respect to a given problem. Let $\Omega_{C}$ be the universe of all possible cases. A case $c \in \Omega_{C}$ is defined as a tuple

$$
c=\left(\mathcal{F}_{c}, \mathcal{D}_{c}, I_{c}\right),
$$

where $\mathcal{F}_{c} \in \Omega_{\mathcal{F}}$ is the set of findings observed in the case $c$. These findings are commonly called problem description. The set $\mathcal{D}_{c} \in \Omega_{\mathcal{D}}$ is the set of diagnoses describing the solution of the case, i.e. which habe been assigned to the state probable. The set $\mathcal{I}_{c}$ contains additional (meta-)information describing the case $c$ in more detail. For the learning task, we consider a case base $C B \subseteq \Omega_{C}$ containing all available cases that have been solved previously.

A simple and intuitive way for representing inferential knowledge is the utilization of diagnostic scores [5]. Then simple scoring rules are applied. 
Definition 1 (Simple Scoring Rule). A simple scoring rule $r$ is denoted as follows:

$$
r=f \stackrel{s}{\rightarrow} d,
$$

where $f \in \Omega_{\mathcal{F}}$ and $d \in \Omega_{\mathcal{D}}$ is the targeted diagnosis. For each rule a symbolic confirmation category $s \in \Omega_{s c r}$ is attached with $\Omega_{s c r} \in\left\{S_{3}, S_{2}, S_{1}, 0, S_{-1}, S_{-2}, S_{-3}\right\}$.

Let $\Omega_{R}$ be the universe of all possible rules for the sets $\Omega_{\mathcal{F}}, \Omega_{\mathcal{D}}$ and $\Omega_{s c r}$. Then, we call $\mathcal{R} \subseteq \Omega_{R}$ the rule base containing the inferential knowledge of the problem domain.

Scores are used to represent a qualitative degree of uncertainty. In contrast to quantitative approaches, e.g. Bayesian methods, symbolic categories state the degree of confirmation or disconfirmation for diagnoses. In this way, a symbolic category $s$ expresses the uncertainty for which the observation of finding $f$ will confirm/disconfirm the diagnosis $d$. Whereas $s \in\left\{S_{1}, S_{2}, S_{3}\right\}$ stand for confirming symbolic categories in ascending order, the categories $s \in\left\{S_{-1}, S_{-2}, S_{-3}\right\}$ are ascending categories for disconfirming a diagnosis. A rule with category 0 has no effect on the diagnosis' state and therefore is usually omitted from the rule base. It is worth noticing, that the value range $\Omega_{s c r}$ of the possible symbolic categories is not fixed. For a more detailed (or coarse) representation of confirmation the value range may be extended (or reduced).

For a given case $c \in \Omega_{C}$ the final state of each diagnosis $d \in \Omega_{\mathcal{D}}$ is determined by evaluating the available scoring rules $r \in \mathcal{R}$ targeting $d$. Thus rules $r=f \stackrel{s}{\rightarrow} d$ contained in $\mathcal{R}$ are activated, iff $f$ is observed in case $c$, i.e. $f \in \mathcal{F}_{c}$. The symbolic categories of the activated rules are aggregated by adding the categories in a way, so that four equal categories result in the next higher category (e.g. $S_{1}+S_{1}+S_{1}+S_{1}=S_{2}$ ) and so that two equal categories with opposite sign nullify (e.g. $S_{1}+S_{-1}=0$ ). For a more detailed or coarse definition of $\Omega_{s c r}$ the aggregation rules may be adapted. A diagnosis is assumed to be probable (i.e. part of the final solution of the case), if the aggregated score is greater or equal than the symbolic category $S_{3}$.

Scoring rules have proven to be useful in large medical knowledge bases, e.g. in the INTERNIST/QMR project [6]7]. For our own work with the shell-kit D3, scores have been proven to be successful in many (large) knowledge system projects, e.g. in a geo-ecological application [8] or in medical domains [3] and technical domains [5] using generalized scores. In context of the PIT system, Fronhöfer and Schramm [9] investigated the probabilistic aspects of scores. The LEXMED [10] project is a successful application developed with the PIT system.

\section{Learning Diagnostic Scores}

In the following we will first discuss diagnostic profiles utilized in the learning method. Then we will briefly discuss necessary data preprocessing steps for the learning task. After that we will outline the method for inductive learning of diagnostic scores from cases.

Diagnostic profiles describe a compact case representation for each diagnosis. For a diagnosis $d \in \Omega_{\mathcal{D}}$ a diagnostic profile $P_{d}=\left(\mathcal{F}_{d}\right.$, frec $\left._{\mathcal{F}}\right)$ contains the findings $\mathcal{F}_{d} \subset \Omega_{\mathcal{F}}$ that occur most frequently with the diagnosis (frequencies stored in frec $_{\mathcal{F}}$ ). 
Learning diagnostic profiles entails, that each profile will initially contain all findings which occur together with the profile's diagnoses. After that we apply a statistical pruning method, such that the profiles contain typical attributes and findings for a given diagnosis. For a more detailled discussion we refer to [11].

The basic algorithm for learning diagnostic scores can only handle discrete valued attributes. Therefore, for handling continuous data we will transform continuous attributes into attributes with discrete partitions in a preprocessing step. For some continuous attributes the expert already defined reasonable partitions. In the case, that there are predefined partitions available, we use these. Otherwise, we used a $k$-means clustering method for discretizing attribute values.

For learning diagnostic scores we first have to identify dependencies between findings and diagnoses. In general, all possible combinations between diagnoses and findings have to be taken into account. However, to reduce the search space, we first learn diagnostic profiles identifying typical findings for a diagnosis. In this way we restrict the set of important findings for a diagnosis using diagnostic profiles.

In summary, we basically apply four steps for learning a diagnostic scoring rule: we first identify a dependency association between a finding $f \in \Omega_{\mathcal{F}}$ and diagnosis $d \in \Omega_{\mathcal{D}}$, rate this dependency, map it to a symbolic category $s \in \Omega_{s c r}$ and finally construct a diagnostic rule: $f \stackrel{s}{\rightarrow} d$.

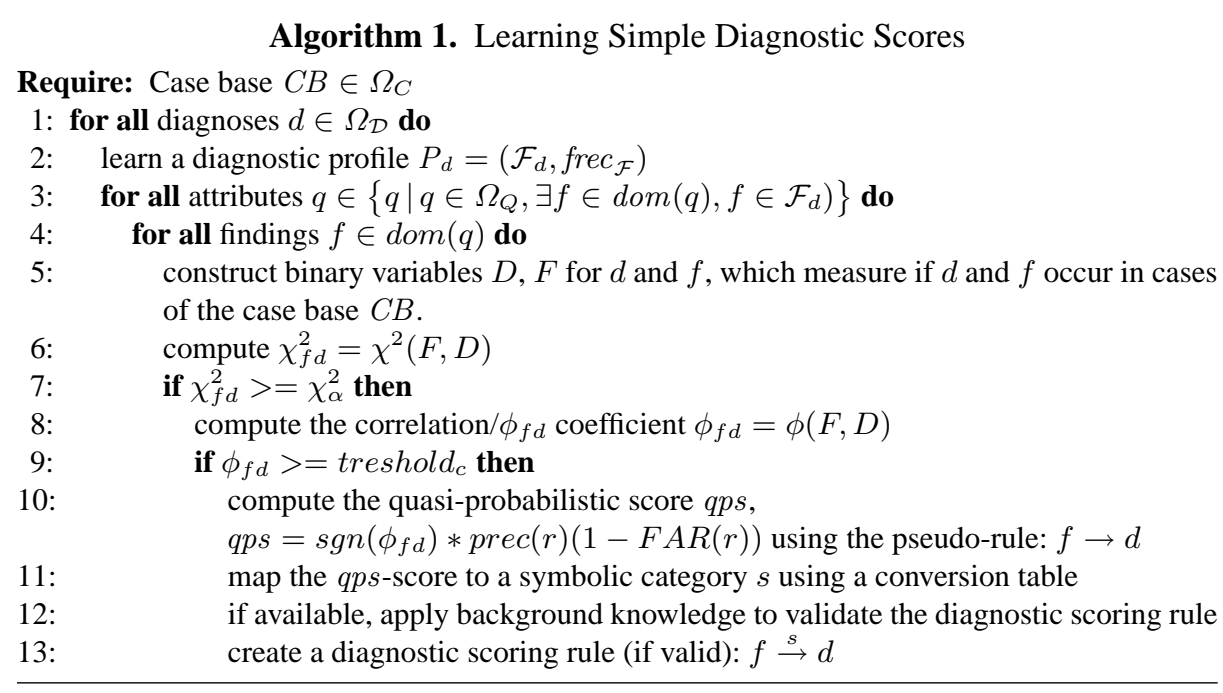

For each diagnosis $d$, we create a diagnostic profile. We consider all attributes (questions) in the profile selecting the findings which are observed in the case base. Then we create a four-fold contingency-table for each finding - diagnosis relation. With the given diagnosis $d$ and finding $f$ of attribute $q$, i.e. $f=q: v$, we construct two binary variables limiting these to cases $\mathcal{C} \subseteq C B$ from the case base in which attribute $q$ is not unknown: a variable $D$ which is true, iff diagnosis $d$ occurs in a case, and false otherwise, and a 
variable $F$ which is true, iff finding $f$ occurs in a case, otherwise $F$ is false likewise. We fill the four-fold table as shown below.

\begin{tabular}{|l|c|c|}
\hline & $D=$ true & $D=$ false \\
\hline$F=$ true & $\mathrm{a}$ & $\mathrm{b}$ \\
$F=$ false & $\mathrm{c}$ & $\mathrm{d}$ \\
\hline
\end{tabular}

The frequency counts denoted in the table are defined as follows:

$$
\begin{aligned}
& a=N(D=\text { true } \wedge F=\text { true }), b=N(D=\text { false } \wedge F=\text { true }), \\
& c=N(D=\text { true } \wedge F=\text { false }), d=N(D=\text { false } \wedge F=\text { false }),
\end{aligned}
$$

where $N($ cond $)$ is the number of times the condition cond is true for cases $c \in \mathcal{C}$. To identify dependencies between findings and diagnoses, we apply the $\chi^{2}$-test for indepence with a certain threshold $\chi_{\alpha}^{2}$ corresponding to confidence level $\alpha$. For binary variables the formula for the $\chi^{2}$-test simplifies to

$$
\chi^{2}(F, D)=\frac{(a+b+c+d)(a d-b c)^{2}}{(a+b)(c+d)(a+c)(b+d)} .
$$

For small sample sizes, we apply the Yates' correction for a more accurate result. For all dependent tuples $(F, D)$ we derive the quality of the dependency using the $\phi$-coefficient

$$
\phi(F, D)=\frac{a d-b c}{\sqrt{(a+b)(c+d)(a+c)(b+d)}},
$$

which measures the degree of association between two binary variables. We use it to discover positive or negative dependencies. If the absolute value of $\phi(F, D)$ is less than a certain threshold threshold $c$, i.e. $|\phi(F, D)|<$ threshold $_{c}$, then we do not consider this weak dependency for rule generation. For the remaining dependencies we generate rules described as follows: If $\phi(F, D)<0$, then we obtain a negative association between the two variables, and we will generate a rule $f \stackrel{s}{\rightarrow} d$ with a negative category $s$. If $\phi(F, D)>0$, then we construct a rule $f \stackrel{s}{\rightarrow} d$ with a positive category $s$.

For determining the exact symbolic confirmation category of the remaining rules $r=f \rightarrow d$, we utilize two measures used in diagnosis: precision and the false alarm rate (FAR), which is also known as the false positive rate, or 1 - specificity. Precision is defined as $\operatorname{prec}(r)=\frac{T P}{T P+F P}$, whereas the false alarm rate is defined as $F A R(r)=$ $\frac{F P}{F P+T N} . T P, T N, F P$ denote the number of true positives, true negatives, and false positives, respectively. These can easily be extracted from the contingency table. For a positive dependency between finding $f$ and $d, T P=a, T N=d$ and $F P=b$. For a negative dependency the situation is different, since we try to predict the absence of the diagnosis, so $T P=b, T N=c$ and $F P=a$. To score the dependency, we first compute a quasi probabilistic score (qps) which we then map to a symbolic category. The numeric qps score is computed as follows:

$$
q p s(r)=\operatorname{sgn}(\phi(D, F)) * \operatorname{prec}(r)(1-F A R(r))
$$

We achieve a tradeoff between the accuracy of the diagnostic scoring rule to predict a disease measured against all predictions and the proportion of false predictions. It 
is worth noting, that often the true positive rate (TPr) (which is also known as recall/sensitivity) is used in combination with the FAR as a measure of accuracy. However, this is mostly applicable to standard rules, which are more complex than diagnostic scores. Since a diagnostic score is a combination of several diagnostic scoring rules, which support each other in establishing a diagnosis, their accuracy needs to be assessed on localized regions of the diagnosis space. So, precision is more suggestive, since it does not take the complete diagnosis space into account, but it measures only the accuracy of the localized prediction. To ease interpretability of the discovered knowledge, we restrict the mapping process to only six different symbolic confirmation categories, three positive and three negative. The $q p s$-scores are mapped to the symbolic categories according to the following conversion table:

\begin{tabular}{|l|lr|}
\hline $\mathbf{q p s}(\mathbf{r}) \quad$ category $(\mathbf{r})$ & $\mathbf{q p s}(\mathbf{r}) \quad$ category $(\mathbf{r})$ \\
\hline$[-1.0,-0.9) \rightarrow S_{-3}$ & $(0.0,0.5) \rightarrow S_{1}$ \\
{$[-0.9,-0.5) \rightarrow S_{-2}$} & {$[0.5,0.9) \rightarrow S_{2}$} \\
{$[-0.5,0.0) \rightarrow S_{-1}$} & {$[0.9,1.0] \rightarrow S_{3}$} \\
\hline
\end{tabular}

We accept the loss of information to facilitate a user-friendly adaptation of the learned diagnostic scores by the expert in a later step.

Including Background Knowledge Sometimes abnormality information about attribute values may be available. We will use these abnormalities, for further shrinking the size of the generated rule base: Let $r=q: v \stackrel{s}{\rightarrow} d$ be a given scoring rule. If $s \in \Omega_{s c r}$ denotes a positive category and $v$ is a normal value of attribute $q$, then we will omit rule $r$, since findings describing normal behavior usually should not increase the confirmation of a diagnosis. Furthermore, if $s$ denotes a negative category and $v$ is an abnormal value of attribute $q$, then we likewise will omit rule $r$, because an abnormal finding usually should not decrease the confirmation of a diagnosis.

As a second type of background knowledge the expert can provide partition class knowledge describing how to divide the set of diagnoses and attributes into partially disjunctive subsets, i.e. partitions. These subsets correspond to certain problem areas of the application domain. For example, in the medical domain of sonography, we have subsets corresponding to problem areas like liver, pancreas, kidney, stomach and intestine. This knowledge is especially useful when diagnosing multiple faults. Since a case may contain multiple solutions, attributes occurring with several diagnoses will be contained in several diagnostic profiles. To give additional support to the dependencies which are discovered, for each such dependency $f \rightarrow d$ we check, if attribute $q$ with $f=q: v$ and diagnosis $d$ are in the same partition class. If the check fails, then we prune the dependency, which reduces noise and irrelevant dependencies.

Diagnostic Scoring Rule Post-Processing To reduce the number of generated rules, and to make them more comprehensible, we merge rules for continuous attributes. We combine rules covering neighboring partitions of attributes into one rule, if they have equal symbolic categories. This does not result in a loss of accuracy, because the rule base is not changed semantically, but only tightened syntactically. 


\section{Evaluation}

We evaluated the presented methods with cases taken from a medical application, which is currently in routine use. The applied SONOCONSULT case base contains 1340 cases, with a mean of diagnoses $M_{d}=4.32 \pm 2.79$ and a mean of relevant findings $M_{f}=$ $76.89 \pm 20.59$ per case.

For the evaluation of our experiments we adopted the commonly used F-measure known from information extraction theory which is appropriate for comparing solutions with multiple faults. For the correct solution $\mathcal{D}_{1}$ and an inferred solution $\mathcal{D}_{2}$ the Fmeasure is defined as follows $\left(D_{1}, D_{2} \subseteq \Omega_{\mathcal{D}}\right)$ :

$$
f\left(\mathcal{D}_{1}, \mathcal{D}_{2}\right)=\frac{\left(\beta^{2}+1\right) \cdot \operatorname{prec}\left(\mathcal{D}_{1}, \mathcal{D}_{2}\right) \cdot \operatorname{recall}\left(\mathcal{D}_{1}, \mathcal{D}_{2}\right)}{\beta^{2} \cdot \operatorname{prec}\left(\mathcal{D}_{1}, \mathcal{D}_{2}\right)+\operatorname{recall}\left(\mathcal{D}_{1}, \mathcal{D}_{2}\right)},
$$

where $\beta$ denotes a constant weight for the precision and $\operatorname{prec}\left(\mathcal{D}_{1}, \mathcal{D}_{2}\right)=\left|\mathcal{D}_{1} \cap \mathcal{D}_{2}\right| /\left|D_{2}\right|$, $\operatorname{recall}\left(\mathcal{D}_{1}, \mathcal{D}_{2}\right)=\left|\mathcal{D}_{1} \cap \mathcal{D}_{2}\right| /\left|\mathcal{D}_{1}\right|$. We used $\beta=1$ for our experiments.

For the evaluation we applied a stratified 10 -fold cross-validation method. We applied two different settings for learning scores, first using no background knowledge for the learning task $(-K)$ and secondly applying all available background knowledge $(+K)$, i.e. abnormality and partition class knowledge. We created several sets of scores depending on the parameter threshold . Two criteria, accuracy and complexity of the learned scores, were used for assessing the quality of the scores. A score is considered to be the more complex, the more findings it contains. This directly corresponds to the number of learned rules per diagnosis (rules/d Mean, with a standard deviation rules/d $S D)$. An overall impression of the complexity of the learned scores is given by the total number of learned rules (rules). Furthermore, as a global complexity measure we count the number of findings/questions which are used in the learning process. Usually a moderate number of findings/questions is considered more comprehensible than a huge number of findings/questions. The results are presented in the following table.

\begin{tabular}{|c|c|c|c|c|c|c|c|c|c|c|c|c|}
\hline treshold $_{c}$ & \multicolumn{2}{|c|}{ rules } & \multicolumn{2}{c|}{ rules/d Mean } & \multicolumn{2}{c|}{ rules/d SD } & \multicolumn{2}{c|}{ questions } & \multicolumn{2}{|c|}{ findings } & \multicolumn{2}{|c|}{ accuracy } \\
\hline & $-K$ & $+K$ & $-K$ & $+K$ & $-K$ & $+K$ & $-K$ & $+K$ & $-K$ & $+K$ & $-K$ & $+K$ \\
\hline 0.10 & 3867 & 956 & 53.71 & 13.27 & 29.54 & 8.43 & 135 & 91 & 403 & 224 & 0.96 & 0.91 \\
0.20 & 2494 & 673 & 34.64 & 9.34 & 19.78 & 5.88 & 135 & 88 & 393 & 193 & 0.96 & 0.90 \\
0.30 & 1692 & 484 & 23.50 & 6.73 & 12.66 & 3.80 & 132 & 83 & 357 & 164 & 0.94 & 0.89 \\
0.40 & 1213 & 364 & 16.85 & 5.06 & 8.72 & 2.49 & 126 & 77 & 307 & 143 & 0.92 & 0.87 \\
0.50 & 890 & 272 & 12.36 & 3.77 & 6.43 & 1.68 & 118 & 72 & 264 & 124 & 0.91 & 0.87 \\
0.60 & 674 & 212 & 9.36 & 2.94 & 4.49 & 1.23 & 111 & 66 & 226 & 110 & 0.86 & 0.81 \\
\hline
\end{tabular}

The high values for the accuracy for low values of threshold $_{c}$ and the large number of rules per diagnosis indicate overfitting of the learned knowledge. This is of course domain dependent, and therefore the expert needs to tune the threshold carefully. With

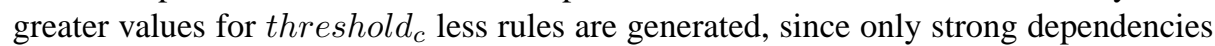
are taken into account. If threshold $d_{c}$ is too high, i.e. if too many rules are pruned, this obviously degrades the accuracy of the learned scores. Applying background knowledge has clearly the effect of strongly reducing the number of scoring rules which are learned, by removing irrelevant and noisy findings from the scoring rules, and thus pruning the scores effectively. Thus, a set of scores with moderate complexity can be obtained, which still yield a sufficient accuracy level. 


\section{Conclusion}

We presented a method for learning simple scoring rules applied for diagnostic tasks. Scoring rules are appealing because of their simplicity and practical relevance in medical decision making. The presented work investigates methods for learning small and simple sets of rules with an acceptable quality concerning diagnostic accuracy and rule complexity. Background knowledge, like abnormalities for attribute values further helped to shrink the size of the rule base, without significantly reducing the accuracy. The evaluation of our methods was implemented using a stratified 10-cross validation applying 1340 cases from a real-life medical application.

In the future, we are planning to improve the presented work by considering subgroups for score extraction, which can focus the scores on significant subspaces of the diagnoses' space. Furthermore, scores can be refined and simplified by aggregating findings into sub-concepts by learning sub-scores first, and using these sub-scores in combination with other findings for the final scores. Another promising approach is the automatic adaptation of thresholds for scores. We expect such ideas to be a good start for significantly improving the combination of accuracy and complexity of the scores.

\section{References}

1. Ohmann, C., et al.: Clinical Benefit of a Diagnostic Score for Appendicitis: Results of a Prospective Interventional Study. Archives of Surgery 134 (1999) 993-996

2. Eich, H.P., Ohmann, C.: Internet-Based Decision-Support Server for Acute Abdominal Pain. Artificial Intelligence in Medicine 20 (2000) 23-36

3. Buscher, H.P., Engler, C., Fuhrer, A., Kirschke, S., Puppe., F.: HepatoConsult: A KnowledgeBased Second Opinion and Documentation System. Artificial Intelligence in Medicine 24 (2002) 205-216

4. Puppe, F.: Knowledge Reuse Among Diagnostic Problem-Solving Methods in the Shell-Kit D3. Int. J. Human-Computer Studies 49 (1998) 627-649

5. Puppe, F., Ziegler, S., Martin, U., Hupp, J.: Wissensbasierte Diagnosesysteme im ServiceSupport. Springer Verlag (2001)

6. R., M., Pople, H.E., Myers, J.: Internist-1, an Experimental Computer-Based Diagnostic Consultant for General Internal Medicine. NEJM 307 (1982) 468-476

7. Pople, H.E.: Heuristic Methods for Imposing Structure on Ill-Structured Problems: The Structuring of Medical Diagnostics. In Szolovits, P., ed.: Artificial Intelligence in Medicine. AAAS, Westview Press (1982)

8. Neumann, M., Baumeister, J., Liess, M., Schulz, R.: An Expert System to Estimate the Pesticide Contamination of Small Streams using Benthic Macroinvertebrates as Bioindicators, Part 2. Ecological Indicators 2 (2003) 391-401

9. Fronhöfer, B., Schramm, M.: A Probability Theoretic Analysis of Score Systems. In KernIsberner, G., Lukasiewicz, T., Weydert, E., eds.: KI-2001 Workshop: Uncertainty in Artificial Intelligence. (2001) 95-108

10. Schramm, M., Ertel, W.: Reasoning with Probabilities and Maximum Entropy: The System PIT and its Application in LEXMED. In et al., K.I., ed.: Operations Research Proceeedings, Springer Verlag (1999) 274-280

11. Baumeister, J., Atzmueller, M., Puppe, F.: Inductive Learning for Case-Based Diagnosis with Multiple Faults. In: Advances in Case-Based Reasoning. Volume 2416 of LNAI. SpringerVerlag, Berlin (2002) 28-42 Proceedings of the 6th European Conference on Case-Based Reasoning (ECCBR-2002). 